\title{
Photoinduced self-stirring crystals caused by gas release
}

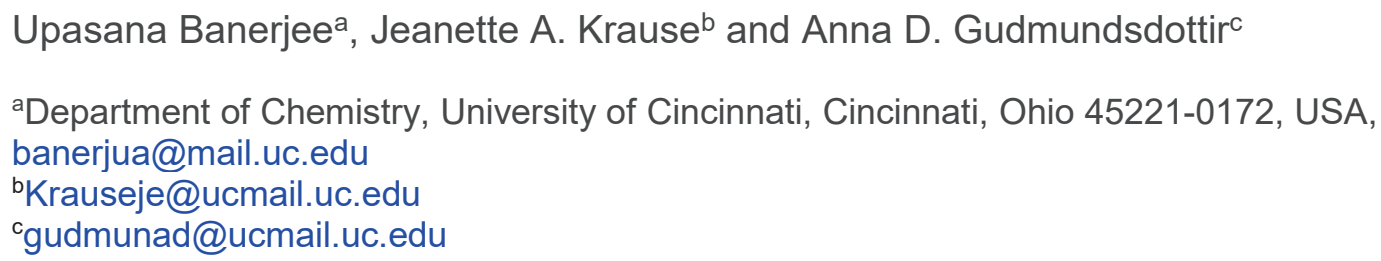

Azido photochemistry has gained a lot of interest recently due to their versatile application in synthesizing pharmaceutically significant heterocycles. We report a sustainable approach of synthesizing pyrrole from dieneazide derivatives using visible light LEDs and sunlight in crystals. Optical microscopy reveals self-stirring photodynamic response of the dieneazide crystals and was correlated with the intermolecular forces inside the crystal lattice to investigate the basis of high photo conversion in the solid-state. 